\title{
Obstructive sleep apnoea and hypertension: the ESADA study
}

\author{
R. Doug McEvoy ${ }^{1,2}$
}

Affiliations: 'Adelaide Institute for Sleep Health, Repatriation General Hospital, Adelaide, Australia. ${ }^{2}$ School of Medicine, Faculty of Medicine, Nursing and Health Sciences, Flinders University, Adelaide, Australia.

Correspondence: R. Doug McEvoy, Adelaide Institute for Sleep Health, Repatriation General Hospital, Daws Rd, Daw Park, 5041, Australia. E-mail: doug.mcevoyahealth.sa.gov.au

@ERSpublications

Patients sent for sleep apnoea assessment show overnight oxygen deprivation is a strong predictor of hypertension http://ow.ly/zub51

Population-based epidemiological studies strongly implicate obstructive sleep apnoea (OSA) as an independent risk factor for cardiovascular morbidity and mortality [1-7]. Final proof of a causal relationship between OSA and premature cardiovascular disease, and the true strength of that relationship, will depend on the results of large, well-designed randomised controlled trials of OSA treatment, several of which are ongoing [8]. In the meantime there is considerable interest in the various mechanistic pathways whereby OSA might lead to increased cardiovascular disease/events. A clearer understanding of these pathways will probably have practical therapeutic implications for reducing cardiovascular risk in OSA populations in the future. Devices such as continuous positive airway pressure (CPAP) and mandibular repositioning devices are effective treatments for OSA, but the reality is that they are found to be unacceptable or are used infrequently by many patients with OSA, particularly those who are minimally symptomatic. Other treatments directed specifically at mitigating cardiovascular risk in such patients may be needed and this will require a clear understanding of the dominant mechanisms for increased cardiovascular disease and/or cardiovascular events in OSA.

Systemic hypertension is the most important risk factor for cardiovascular disease. There is now strong evidence from randomised controlled trials of CPAP treatment of OSA that sleep apnoea is responsible for an increase in nocturnal and diurnal blood pressure, although to what extent OSA causes clinical hypertension is less certain. While early cross sectional data from community studies found a relatively robust independent association between OSA and prevalent hypertension [9-11], the results of longitudinal studies examining the role of OSA in the development of new hypertension have been less convincing. Some studies have shown an independent association between OSA and incident hypertension [12, 13], while others have not $[14,15]$. Also, randomised controlled trials of CPAP treatment in OSA have shown only relatively small reductions in blood pressure $(\sim 2-4 \mathrm{mmHg})$, with the treatment effect size being remarkably similar between OSA patients with and without hypertension [16-19]. Nevertheless, the blood pressure increase attributable to OSA is likely to increase the risk of major cardiovascular events by $\sim 7 \%$ [20], which is a significant public health concern when one considers the relatively high and rising prevalence of OSA in the community [21], and the fact that most disease is currently undiagnosed.

Apnoea and hypopnoea events are followed by sudden arousal from sleep and transient hyperpnoea. These changes result in acute surges in blood pressure during the night in OSA patients but they also induce a number of persistent, inter-related neurophysiological and biochemical changes [22, 23], which alter central neural control of blood pressure and increase peripheral arterial smooth muscle tone such that blood pressure levels are also increased in the daytime. Decreased baroreflex sensitivity and increased chemoreceptor sensitivity combine to increase the blood pressure set point and increase background

Received: July 162014 | Accepted: July 162014

Conflict of interest: Disclosures can be found alongside the online version of this article at erj.ersjournals.com

Copyright @ERS 2014 
arterial sympathetic nerve activity [24]. Circulating levels of vasoconstrictor peptides, such as endothelin-1 and angiotensin II, are increased and the level of circulating nitric oxide, a potent vasodilator, is decreased. Vascular endothelial dysfunction also probably contributes to increased smooth muscle vascular tone [25]. Reactive oxygen species [26], endothelin-1 and angiotensin II probably play a key role in modulating baroand chemoreflex pathways and increasing sympathetic outflow [27].

There are a number of acute pathophysiological events during cyclical sleep apnoea/hypopnoea that could contribute to these changes. The two that come most readily to mind are easiest to measure during human sleep studies and that have been subjected to animal experimentation are intermittent hypoxia and sleep arousal/fragmentation. However, other components of the apnoea/hypopnoea-arousal cycle, such as intermittent hypercapnia, large intrathoracic negative pressure swings and perhaps the extent of postarousal re-oxygenation and hypocapnia, could also alter baroreceptor, chemoreceptor and endothelial function. It also appears that snore vibrations can cause direct damage to the carotid arteries [28], which means that post-apnoeic snores or snorts themselves could have the potential to affect carotid baro- and chemoreceptor function [29].

Knowing which mechanism(s) are primarily responsible for raising blood pressure in OSA could have direct clinical utility. Reversal of apnoea with CPAP or weight loss would be the best "catch all" treatment but these therapeutic approaches often fail because of poor patient adherence. If intermittent hypoxia was the dominant mechanism supplemental oxygen could be a useful alternative treatment in selected OSA cases (e.g. those with pharmacologically resistant hypertension). Conversely, if arousals, cyclical hypercapnia/ hypocapnia and mechanical phenomena (e.g. large intrathoracic negative pressure swings or snore vibrations) were stronger drivers of the blood pressure changes, alternative approaches would be needed.

Animal experiments have been employed in an attempt to address some of these questions. Experiments in rodents have shown that intermittent hypoxia over several days to weeks can induce sustained hypertension, largely by an upregulation of sympathetic nervous system activity [30], and have been helpful in delineating the molecular mechanisms responsible for this [27]. More recently, substantial elevations in daytime and night-time blood pressure have been shown in humans after 2 weeks of experimental night-time intermittent hypoxia [31]. Previously, a dog model showed that 1-3 months of repetitive upper airway obstructions during sleep increased night-time and daytime blood pressure whereas repetitive tone-induced arousals caused an elevation in night-time blood pressure alone [32].

In this issue of the European Respiratory Journal, TKACOVA et al. [33] report cross sectional findings on OSA and hypertension in $\sim 12000$ patients (70\% male) attending a sleep clinic for diagnosis of suspected OSA in 24 sleep centres from six European countries and Israel between 2007 and 2013. It is one of the first reports emanating from the large European Sleep Apnoea Database (ESADA) cohort study set up in 2005 as part of the European Union Cooperation in Science and Technology (COST) Action B26 programme. To maximise pan-European participation and ensure a large sample size, a pragmatic approach was used in setting up the ESADA study such that laboratories were able to keep their preferred mode of sleep study recording and scoring. The authors report a strong association between OSA and patient reports of doctordiagnosed hypertension (regardless of medication prescription) after controlling for known or possible confounders, i.e. age, sex, body mass index, neck circumference, type 2 diabetes, dyslipidaemia and previous smoking. The odds ratio for hypertension in the upper quartile of the apnoea/hypopnoea index (AHI $\geqslant 39$ ) compared to the lowest quartile (AHI <6) was 1.51 (95\% CI 1.31-1.73; $<<0.0001$ for trend across the four quartiles). A similar strong, independent association was found between oxygen desaturation index (ODI) (reported by laboratories as either $\geqslant 3 \%$ or $\geqslant 4 \%$ ) and hypertension. Sensitivity analyses examining the relationship between AHI and ODI using a stricter definition of hypertension (i.e. hypertension considered present only if patients were on anti-hypertensive medication) produced similar results. The results of this part of the study are clear cut and add to the growing body of evidence implicating OSA as a cause of hypertension. A particular strength of this study is the large sample size, which has been drawn from diverse ethnic and sociocultural settings across Europe.

The investigators then attempted to use their data to explore possible mechanisms for OSA-associated hypertension. When AHI and ODI were simultaneously entered into a multiple logistic regression model it was found that only ODI remained a predictor of prevalent hypertension. AHI provided no independent predictive value and was rejected from the model. They concluded that for practical considerations ODI may, therefore, be the preferred method for assessing risk of OSA-related hypertension and cardiovascular disease. Indeed, if this result is confirmed by longitudinal studies of incident hypertension and cardiovascular disease in the ESADA cohort, it may well enable routine screening sleep studies to be simplified and costs reduced. The investigators then speculated that, based on their data, intermittent hypoxia was likely to be the dominant factor in the development of hypertension in OSA, rather than other pathophysiological mechanisms associated with OSA (e.g. sleep fragmentation and intermittent 
hypercapnia). A problem with this interpretation is that AHI involves manual scoring of polygraph or polysomnography recordings that can be highly variable both between and within observers, whereas ODI, which is calculated using proprietary software, is very reproducible. Therefore, one is left wondering whether the stronger relationship shown between ODI and hypertension relates more to methodological differences, and not as TKACOVA et al. [33] suggest, that oxygen desaturation is a stronger driver of hypertension in OSA than other pathophysiological characteristics of the disorder. The diverse range of sleep study recording techniques and AHI scoring methods used by ESADA sites (detailed in the supplementary material of TKACOVA et al. [33]) adds to this concern. While the large sample size enabled the authors to conduct a number of sensitivity analyses to try to take account of these methodological issues, unless recording and scoring methods are very carefully standardised, AHI remains a highly variable metric and limits the value of this sort of analysis.

Multiple studies using experimental intermittent hypoxia lasting days to weeks in animal models, and now in humans, have demonstrated sustained secondary increases in blood pressure. These acute proof-ofconcept experiments and the various follow-up studies that have examined the biochemical correlates of blood pressure changes in these models add credence to the theory that intermittent hypoxia plays an important role in OSA-associated hypertension. It must be remembered, however, that intermittent hypoxia also fragments sleep and the extent of sleep fragmentation, independent of severity of desaturation, has been associated with hypertension in OSA [34]. Finally, to add fuel to this debate it has been shown in a recent three-way 12-week randomised trial comparing oxygen and CPAP treatments for OSA with lifestyle advice (i.e. control group), that CPAP lowered blood pressure while oxygen did not [35].

Further research is required to fully unravel the pathways and mechanisms by which blood pressure is increased in OSA patients. Because of its very large size, the ESADA collaboration may be able to contribute significantly to this endeavour. For example, it may have sufficient subjects to conduct case-control studies that compare blood pressure between OSA patients who have significant sleep fragmentation but little to no oxygen desaturation versus patients with the same AHI and arousal index but marked oxygen desaturation, or between OSA groups matched for AHI and oxygen desaturation but with different levels of sleep fragmentation.

\section{References}

1 Somers VK, White DP, Amin R, et al. Sleep apnea and cardiovascular disease: an American Heart Association/ American College of Cardiology Foundation Scientific Statement from the American Heart Association Council for High Blood Pressure Research Professional Education Committee, Council on Clinical Cardiology, Stroke Council, and Council on Cardiovascular Nursing. J Am Coll Cardiol 2008; 52: 686-717.

2 Gottlieb DJ, Yenokyan G, Newman AB, et al. Prospective study of obstructive sleep apnea and incident coronary heart disease and heart failure: the sleep heart health study. Circulation 2010; 122: 352-360.

3 Yaggi HK, Concato J, Kernan WN, et al. Obstructive sleep apnea as a risk factor for stroke and death. N Engl J Med 2005; 353: 2034-2041.

4 Redline S, Yenokyan G, Gottlieb DJ, et al. Obstructive sleep apnea-hypopnea and incident stroke: the sleep heart health study. Am J Respir Crit Care Med 2010; 182: 269-277.

5 Young T, Finn L, Peppard PE, et al. Sleep disordered breathing and mortality: eighteen-year follow-up of the Wisconsin sleep cohort. Sleep 2008; 31: 1071-1078.

6 Marshall NS, Wong KK, Liu PY, et al. Sleep apnea as an independent risk factor for all-cause mortality: the Busselton Health Study. Sleep 2008; 31: 1079-1085.

7 Punjabi NM, Caffo BS, Goodwin JL, et al. Sleep-disordered breathing and mortality: a prospective cohort study. PLoS Med 2009; 6: e1000132.

8 Gottlieb DJ, Craig SE, Lorenzi-Filho G, et al. Sleep apnea cardiovascular clinical trials - current status and steps forward: the International Collaboration of Sleep Apnea Cardiovascular Trialists. Sleep 2013; 36: 975-980.

9 Young T, Peppard P, Palta M, et al. Population-based study of sleep-disordered breathing as a risk factor for hypertension. Arch Intern Med 1997; 157: 1746-1752.

10 Bixler EO, Vgontzas AN, Lin HM, et al. Association of hypertension and sleep-disordered breathing. Arch Intern Med 2000; 160: 2289-2295.

11 Nieto FJ, Young TB, Lind BK, et al. Association of sleep-disordered breathing, sleep apnea, and hypertension in a large community-based study. Sleep Heart Health Study. JAMA 2000; 283: 1829-1836.

12 Peppard PE, Young T, Palta M, et al. Prospective study of the association between sleep-disordered breathing and hypertension. N Engl J Med 2000; 342: 1378-1384.

13 Marin JM, Agusti A, Villar I, et al. Association between treated and untreated obstructive sleep apnea and risk of hypertension. JAMA 2012; 307: 2169-2176.

14 Cano-Pumarega I, Duran-Cantolla J, Aizpuru F, et al. Obstructive sleep apnea and systemic hypertension: longitudinal study in the general population: the Vitoria Sleep Cohort. Am J Respir Crit Care Med 2011; 184: 1299-1304.

15 O'Connor GT, Caffo B, Newman AB, et al. Prospective study of sleep-disordered breathing and hypertension: the Sleep Heart Health Study. Am J Respir Crit Care Med 2009; 179: 1159-1164.

16 Martinez-Garcia MA, Capote F, Campos-Rodriguez F, et al. Effect of CPAP on blood pressure in patients with obstructive sleep apnea and resistant hypertension: the HIPARCO randomized clinical trial. JAMA 2013; 310: $2407-2415$. 
Barbe F, Duran-Cantolla J, Capote F, et al. Long-term effect of continuous positive airway pressure in hypertensive patients with sleep apnea. Am J Respir Crit Care Med 2010; 181: 718-726.

18 Duran-Cantolla J, Aizpuru F, Montserrat JM, et al. Continuous positive airway pressure as treatment for systemic hypertension in people with obstructive sleep apnoea: randomised controlled trial. BMJ 2010; 341 : c5991.

19 Montesi SB, Edwards BA, Malhotra A, et al. The effect of continuous positive airway pressure treatment on blood pressure: a systematic review and meta-analysis of randomized controlled trials. J Clin Sleep Med 2012; 8: 587-596.

20 Turnbull F, Neal B, Ninomiya T, et al. Effects of different regimens to lower blood pressure on major cardiovascular events in older and younger adults: meta-analysis of randomised trials. BMJ 2008; 336: 1121-1123.

21 Peppard PE, Young T, Barnet JH, et al. Increased prevalence of sleep-disordered breathing in adults. Am J Epidemiol 2013; 177: 1006-1014.

22 Phillips CL, O’Driscoll DM. Hypertension and obstructive sleep apnea. Nat Sci Sleep 2013; 5: 43-52.

23 Prabhakar NR, Kumar GK. Mechanisms of sympathetic activation and blood pressure elevation by intermittent hypoxia. Respir Physiol Neurobiol 2010; 174: 156-161.

24 Somers VK, Dyken ME, Clary MP, et al. Sympathetic neural mechanisms in obstructive sleep apnea. J Clin Invest 1995; 96: 1897-1904

25 Kato M, Roberts-Thomson P, Phillips BG, et al. Impairment of endothelium-dependent vasodilation of resistance vessels in patients with obstructive sleep apnea. Circulation 2000; 102: 2607-2610.

26 Lavie L, Vishnevsky A, Lavie P. Evidence for lipid peroxidation in obstructive sleep apnea. Sleep 2004; 27: 123-128.

27 Sunderram J, Androulakis IP. Molecular mechanisms of chronic intermittent hypoxia and hypertension. Crit Rev Biomed Eng 2012; 40: 265-278.

28 Lee SA, Amis TC, Byth K, et al. Heavy snoring as a cause of carotid artery atherosclerosis. Sleep 2008; 31: 1207-1213.

29 Schobel C, Fietze I, Glos M, et al. Nocturnal snoring decreases daytime baroreceptor sensitivity. Respir Med 2014; 108: 1049-1055.

30 Bao G, Metreveli N, Li R, et al. Blood pressure response to chronic episodic hypoxia: role of the sympathetic nervous system. J Appl Physiol (1985) 1997; 83: 95-101.

31 Tamisier R, Pèpin JL, Rèmy J, et al. 14 nights of intermittent hypoxia elevate daytime blood pressure and sympathetic activity in healthy humans. Eur Respir J 2011; 37: 119-128.

32 Brooks D, Horner RL, Kozar LF, et al. Obstructive sleep apnea as a cause of systemic hypertension. Evidence from a canine model. J Clin Invest 1997; 99: 106-109.

33 Tkacova R, McNicholas WT, Javorsky M, et al. Nocturnal intermittent hypoxia predicts prevalent hypertension in the European Sleep Apnoea Database cohort study. Eur Respir J 2014; 44: 931-941.

34 Noda A, Yasuma F, Okada T, et al. Influence of movement arousal on circadian rhythm of blood pressure in obstructive sleep apnea syndrome. J Hypertens 2000; 18: 539-544.

35 Gottlieb DJ, Punjabi NM, Mehra R, et al. CPAP versus oxygen in obstructive sleep apnea. N Engl J Med 2014; 370 : 2276-2285. 